\title{
Topical treatment of life threatening haemoptysis from aspergillomas
}

\author{
M Rumbak, G Kohler, C Eastrige, H Winer-Muram, M Gavant
}

\begin{abstract}
Background - The efficacy of topical treatment of 12 episodes of severe life threatening haemoptysis from a pulmonary aspergilloma in 11 patients is reviewed. Methods - A retrospective review was performed on five white and six AfricanAmerican patients of mean age 49 years. The underlying diseases were bronchiectasis, sarcoidosis, tuberculosis, or histoplasmosis. The patients were prospectively considered poor surgical risks because they had a forced expiratory volume in one second $\left(\mathrm{FEV}_{1}\right)$ of $<50 \%$ predicted and an arterial oxygen tension $\left(\mathrm{PaO}_{2}\right)$ of $<7.95 \mathrm{kPa}$ breathing room air. As surgical intervention was not possible, local intracavitary instillation of sodium or potassium iodide was performed. Two patients were previously treated with amphotericin B. In one patient sodium iodide alone was used and in the remaining eight potassium iodide alone was instilled. The transcricothyroid approach was used in six patients and the percutaneous approach in five.
\end{abstract}

Results - Haemoptysis ceased within 72 hours in all patients after the instillation of sodium or potassium iodide. There was no morbidity or mortality, and side effects included slight irritation on instillation of the iodide solution and occasional cough which was easily controlled. All patients were alive at least one year later.

Conclusion - Intracavitary treatment is a viable option in the poor risk patient with life threatening haemoptysis from an aspergilloma.

(Thorax 1996;51:253-255)

Keywords: haemoptysis, Aspergillus, topical treatment.

Haemoptysis is the most common complication of a pulmonary aspergilloma occurring in up to $74 \%$ of cases. ${ }^{1}$ Massive haemoptysis ( $>600 \mathrm{ml} / 24$ hours) has been fatal in $28 \%$ of these patients. ${ }^{2}$ Surgical resection remains the treatment of choice for patients with adequate pulmonary reserve. ${ }^{12}$ Even in cases with a good pulmonary reserve, $5 \%$ of patients in one series died of complications after surgery and $15 \%$ of the survivors developed significant haemorrhage and bronchopleural fistulae. ${ }^{3}$ There are many patients whose general medical condition precludes surgical resection and topical treatment with various drugs has been attempted. The success of these treatments has been only moderate in patients both with and without severe haemoptysis. ${ }^{1-11}$ We present our ex- perience with topical treatment of aspergillomas and massive life threatening haemoptysis over a 22 year period.

\section{Methods}

PATIENTS

The case records of 11 patients who presented between 1971 and 1993 with 12 episodes of severe haemoptysis from an aspergilloma were retrospectively reviewed. Their ages ranged from 31 to 60 years (mean 49). There were five white and six African-American patients, all of whom were men. The causes of the underlying lung disease were sarcoidosis, tuberculosis, histoplasmosis, or bronchiectasis. Each patient had an aspergilloma documented on a chest radiograph or computed tomographic (CT) scan. Aspergillus cultures and serum precipitins were positive. The patients were prospectively deemed unsuitable for resection due to poor pulmonary reserve with a forced expiratory volume in one second $\left(\mathrm{FEV}_{1}\right)$ of $<50 \%$ predicted and an arterial oxygen tension $\left(\mathrm{PaO}_{2}\right)$ of $<7.95 \mathrm{kPa}$ breathing room air. All patients had presented with haemoptysis of $>600 \mathrm{ml} / 24$ hours and had required transfusions to maintain their arterial systolic blood pressure above $100 \mathrm{~mm} \mathrm{Hg}$. They underwent bronchoscopy to confirm the site of the haemorrhage and to exclude other causes of bleeding. The underlying disease that predisposed to cavitary lung disease, the route of instillation, the substance used, the affected lobe, and the outcome are summarised in the table.

\section{TECHNIQUES}

Five patients (nos 1, 3, 4, 5 and 9) were treated percutaneously. They were pretreated with $0.6 \mathrm{mg}$ atropine intramuscularly, $2 \%$ lignocaine was used to anaesthetise the site, and a thin walled 18 gauge needle was passed into the cavity under fluoroscopic guidance. A small amount of water soluble iodinated contrast medium was instilled to confirm that the needle tip was in the cavity. Using standard Seldinger catheterisation methods, an angiographic guide wire was passed through the needle and coiled in the cavity. A $5 \mathrm{Fr} 30 \mathrm{~cm}$ long, multi sidehole catheter (Cook, Illinois, USA) was then advanced into the cavity, capped, and fixed in place with a retention suture.

A modification of the bronchoscopic technique via a transcricothyroid membrane approach was employed in four patients (nos 2, 6,7 , and 8). Premedication with intramuscular atropine was again used. Oxygen was delivered at $2-41 / \mathrm{min}$ by nasal cannula and sedation was 
Characteristics of patients with massive life threatening haemoptysis from aspergilloma

\begin{tabular}{|c|c|c|c|c|}
\hline Patient no. & Underlying disease & Route of application & Lobe & Drug \\
\hline 1 & Tuberculosis & Percutaneous & Right upper & $\begin{array}{l}\text { Amphotericin B + } \\
\text { sodium iodide\# }\end{array}$ \\
\hline 2 & Tuberculosis & Transcricothyroid & Right upper & $\begin{array}{l}\text { Amphotericin B+ } \\
\text { potassium iodide }\end{array}$ \\
\hline 3 & Tuberculosis & Percutaneous & Left upper & Potassium iodide \\
\hline 4 & Bronchiectasis & Percutaneous & Left upper & Potassium iodide \\
\hline 5 & Tuberculosis & Percutaneous & Right upper & Potassium iodide \\
\hline 6 & Histoplasmosis & Transcricothyroid & Right upper & Potassium iodide \\
\hline 7 & Sarcoidosis & Transcricothyroid & Left upper & Potassium iodide \\
\hline 8 & Tuberculosis & Transcricothyroid & Right upper & Potassium iodide \\
\hline 9 & Tuberculosis & Percutaneous & Right upper & Sodium iodide \\
\hline 10 & Tuberculosis & Transcricothyroid & Right upper & Potassium iodide \\
\hline $11 a^{*}$ & Sarcoidosis & Transcricothyroid & Right upper & Potassium iodide\# \\
\hline $11 b^{*}$ & Sarcoidosis & Transcricothyroid & Left upper & Potassium iodide\# \\
\hline
\end{tabular}

* Patient 11 had two episodes of life threatening haemoptysis (11a and b). These episodes took place one year apart.

\# Disappearance of aspergilloma on CT scan or chest radiograph.

achieved with 1-2 mg Versed (Roche, Switzerland) intravenously. After a standard sterile preparation and local anaesthetic, a thin walled 18 guage needle was advanced under manual palpation through the cricothyroid membrane. A $1 \mathrm{~mm}$ angiographic guidewire was directed through the needle towards the distal trachea. A 5 Fr 50-60 cm multi sidehole catheter with a $45^{\circ}$ angled distal curve (Cook, Illinois, USA) was advanced over the wire to the carina and into the target mainstem bronchus. Using small injections of the contrast medium, the wire and catheter were placed in the segmental bronchus under fluoroscopic guidance and ultimately coiled in the cavity. The position of the catheter tip was confirmed and the catheter was capped and fixed to the skin at the entry site.

The airways are often distorted and negotiating the catheter into the correct segment or subsegment under direct vision may be easier. Any route is acceptable provided the operator is experienced. In the last three episodes in two patients the following technique was used. The aspergilloma was identified using CT scanning and percutaneous transcricothyroidotomy was performed using a modified Seldinger technique. The needle of the pulmonary artery catheter introducer kit (Cordis, Baxter, Santa Ana, California, USA) was inserted into the trachea through the cricothyroid membrane and the guidewire was introduced into the lumen of the trachea through the needle. The catheter was withdrawn leaving the guidewire in the lumen of the trachea. The dilator was threaded on to the wire and used to dilate the hole in the cricothyroid membrane. After the dilator was withdrawn, the Cordis was fed over the wire into the lumen of the trachea and the wire was then withdrawn leaving the Cordis in situ. A single loop suture was placed through the distal end of a $5 \mathrm{Fr}$ angiocatheter which was then inserted through the Cordis into the trachea. The bronchoscope was passed into the trachea from above. A pair of biopsy forceps was inserted through the working channel of the bronchoscope and its jaws closed around the suture at the distal tip of the angiocatheter. Both were advanced into the segment in which the aspergilloma was situated. The bronchoscope was then withdrawn and the position of the angiocatheter was confirmed on a CT scan and secured with a suture at the skin.
The patient was placed with the cavity dependent and $2 \mathrm{ml}$ of $2 \%$ lignocaine were given before the first instillation of sodium or potassium iodide to prevent local irritation. A few minutes later $5 \mathrm{ml}$ of a $2 \%$ solution (diluted with normal saline) of potassium iodide (Roxane, USA) or sodium iodide (Malinkrodt, USA) was instilled into the cavity. The angiocatheter was then flushed with an additional $5 \mathrm{ml}$ of air to ensure that no residual drug remained in the catheter. The patients were rotated between upright, supine, and decubitus positions for 15 minutes each. The entire procedure was repeated three times a day. After four days in hospital the treatment was completed on an outpatient basis for a total of 5-6 $g$ potassium iodide (15-20 days).

In the two patients who received amphotericin B the following procedure was used. The cavity was cannulated percutaneously as described above and $2 \mathrm{ml}$ of $1 \%$ lignocaine was injected into the cavity followed five minutes later by an initial dose of $5 \mathrm{mg}$ amphotericin B. On the following day this was increased to $50 \mathrm{mg}$ in $10 \mathrm{ml} 5 \%$ dextrose given as a once daily dose. Patient 1 received amphotericin B for three days and patient 2 for four days.

\section{Results}

The haemoptysis stopped within 72 hours of treatment with both sodium and potassium iodide. The first two patients had been previously treated with amphotericin $\mathbf{B}$ by the percutaneous or transcricothyroid routes, respectively (table). Patient no. 11 presented on two different occasions with severe haemoptysis and treatment was successfully performed in two different areas of the lung. Resolution of the signs of the aspergilloma on the chest radiograph occurred in only three patients at four, six, and seven weeks after the beginning of treatment. Major side effects did not occur. Minor coughing due to local irritation was associated with the instillation of potassium iodide in one subject. This was prevented in the others with local instillation of lignocaine before the iodide solution. Coughing after instillation of the antifungal treatment occurred in five patients but was easily controlled with a short course of codeine. All patients were alive at one year and four at five years. One died at two years, two at three years, and two at four years after the haemorrhage. The cause of death in all the known cases was respiratory failure. The other two patients were lost to follow up.

\section{Discussion}

Due to the morbidity and mortality in patients with poor lung function who undergo surgery, other methods of treatment have been attempted for severe haemoptysis from an aspergilloma. Bronchial arterial embolisation which is effective for patients with lung cancer, tuberculosis, and cystic fibrosis has been disappointing in patients with aspergillomas. ${ }^{6}$ The cavities have a rich blood supply from the bronchial, axillary, and subclavian arteries and 
this extensive network does not favour embolisation. The spinal arteries may also be occluded during the embolisation. ${ }^{6}$

In studies in which massive haemoptysis was not the sole reason for using topical treatment, the results have been mixed with success reported in four of $\operatorname{six}^{10}$ and in 11 of 20 patients. ${ }^{11}$ In one study slightly better initial results were found in four of five patients. All these patients tolerated the procedure well and some completed their courses of treatment as outpatients. Symptoms resolved immediately but only two patients remained free of cough and mild haemoptysis after eight and 12 months respectively. ${ }^{5}$

In our series of patients with massive haemoptysis the haemoptysis resolved permanently and consistently within 72 hours. Others have reported similar results in response to topical treatment in patients with severe haemorrhage. ${ }^{612}$ Amphotericin B, N-acetylcysteine, and aminocaproic acid instilled topically have successfully halted the severe haemoptysis in six episodes in four patients. ${ }^{6}$ Severe haemoptyisis in one patient was controlled when aminocaproic acid was instilled into the cavity at the bleeding site after thoracotomy failed to control the haemorrhage. ${ }^{12}$

Possible mechanisms by which aspergillomas produce haemoptysis include the mechanical action on a vascular wall, ${ }^{13}$ elaboration of fungal toxins or enzymes, ${ }^{14}$ or an inflammatory type II antibody antigen injury. ${ }^{15}$ Sclerosis of vessels, drug induced $\mathrm{pH}$ changes in the cavity, direct antifungal activity, and aeration of a previously anaerobic cavity are possible mechanisms for controlling severe haemoptysis. The aspergilloma disappeared radiologically in only three of 12 cases and this may have been spontaneous. ${ }^{1617}$ Since others have described similar findings, ${ }^{56}$ we believe mechanical factors to be more important in the control of haemoptysis than antifungal treatment. However, an antifungal role for the iodides cannot be excluded.

To place this experience in the context of our thoracic unit, a brief summary is given of the patients who had massive haemoptysis from an aspergilloma and whose lung function was sufficient to undergo surgery $\left(\mathrm{FEV}_{1}\right.$ and maximum voluntary ventilation $>50 \%$ predicted, $\mathrm{PaO}_{2}>7.95 \mathrm{kPa}$ ). In the same time period 41 patients underwent surgery; 22 had the aspergilloma resectioned, 14 had lobectomies, four had segmentectomies, one had pneumonectomy, two had wedge resections, and two had thoracoplasties after resection of pleural aspergillomas. The latter had developed bron- chopleural fistulae and presented with severe haemoptysis.

In conclusion, the choice of treatment for a patient with a pulmonary aspergilloma and poor pulmonary reserve who develops haemoptysis remains controversial. Massive haemoptysis needs treatment. We feel that intracavitary treatment through the percutaneous or endobronchial route is a viable choice in such patients. Our preference is for the use of iodides although amphotericin B may be substituted. Clearing of the radiograph occurred in only a few cases and may be spontaneous. ${ }^{1819}$ The exact length of treatment therefore requires further study. The mortality and morbidity of intracavitary treatment is much better in patients with poor respiratory reserve than in those who have a good reserve and undergo surgery. ${ }^{3}$ A prospective study is needed to compare surgery with intracavitary medication in patients with good underlying lung function who develop severe haemoptysis.

1 Glimp RA, Bayer AS. Pulmonary aspergilloma: diagnostic and therapeutic considerations. Arch Intern Med 1983;143: and therap 8 .

2 Eastridge CE, Young JM, Cole F, Gourley MD, Pate JW. Pulmonary aspergillosis. Ann Thorac Surg 1972;13:397403.

3 Jewkes J, Kay PH, Paneth M, Citron KM. Pulmonary aspergilloma: analysis of prognosis in relation to haemoptysis and survey of treatment. Thorax 1983;38:572-8.

4 Yamada H, Kohno S, Koga H, Maesaki S, Kaku M. Topica treatment of aspergilloma by antifungals: relationship between duration of disease and efficacy of therapy. Chest 1993;103:421-5.

5 Jackson M, Flower CDR, Shneerson JM. Treatment of symptomatic aspergillomas with intracavitary instillation of amphotericin B through an indwelling catheter. Thorax of amphotericin B

6 Shapiro MJ, Albelda SM, Mayock RM, McClean GK. Severe hemoptysis associated with pulmonary aspergilloma: percutaneous intracavitary treatment. Chest 1988; 94:1225-31.

7 Hamamoto T, Watanabe K, Ikemoto H. Endobronchial miconazole for pulmonary aspergilloma. Ann Intern Med 1974;98:1030-1.

8 Ramirez RJ. Pulmonary aspergilloma: endobronchial treatment. N Engl f Med 1964;271:1281-5.

9 Adelson HT, Malcom JA. Endocavitary treatment of pulmonary mycetomas. Am Rev Respir Dis 1968;98:87-92.

10 Hargis JL, Bone RC, Stewart J, Rector N, Hiller FC. Intracavitary amphotericin $B$ in the treatment of symptomatic pulmonary aspergillomas. Am $f \mathrm{Med} 1980 ; 60$ : tomatic

11 Krackowka P, Traczyk K, Walczyk K, Halwey H, Elsner Z, Pawlicka L. Local treatment of aspergilloma of the lung with paste containing nystatin or amphotericin B. Tubercle 1970;51:184-91.

12 Fernandez NA. Intracavitary aminocaproic acid for massive pulmonary hemorrhage. Chest 1984;85:839-40.

13 Pimental JC. Pulmonary calcification in the turner-like form of pulmonary aspergillosis: pulmonary aspergilloma. $\mathrm{Am}$ Rev Respir Dis 1966;68:208-16.

14 Campbell MJ, Clayton YM. Bronchopulmonary aspergillosis: a correlation of the clinical and laboratory findings in 272 patients investigated for bronchopulmonary asperin 272 patients investigated for bronchopulm
gillosis. Am Rev Respir Dis 1964;89:186-96.

15 Hilvering C, Stevens EAM, Orie NGM. Fever in aspergillus mycetoma. Thorax 1970;25:19-24.

16 Varkey B, Rose HD. Pulmonary aspergilloma - a rational approach to treatment. $A m$ F Med 1976;61:623-31.

17 Hammerman KJ, Christianson CS, Huntington I. Spontaneous lysis of aspergillomata. Chest 1973;64:697-9.

18 Israel HL, Lencher GS, Atkinson GW. Sarcoidosis and aspergilloma: the role of the surgeon. Chest 1982;32:430-2.

9 Hammerman KJ, Sarosi GA, Tosh FF. Amphotericin B in the treatment of saprophytic forms of pulmonary aspergillosis. Am Rev Respir Dis 1974;109:57-62. 